\title{
SISTEMA $W E B$ PARA MODELAGEM MOLECULAR TRIDIMENSIONAL UTILIZANDO REALIDADE AUMENTADA COMO RECURSO INOVADOR DE ENSINO E DE DEMOCRATIZAÇÃO DA INFORMAÇÃO BIOTECNOLÓGICA EM REDE
}

\author{
Alex Eder da Rocha Mazzuco ${ }^{1,3}$, Aliane Loureiro Krassmann ${ }^{2}$, Denis da Silva \\ Garcia $^{3}$, Giliane Bernardi ${ }^{1}$ \\ ${ }^{1}$ Programa de Pós-Graduação em Tecnologias Educacionais em Rede - PPGTER - \\ Universidade Federal de Santa Maria (UFSM) - Santa Maria - RS. \\ ${ }^{2}$ Programa de Pós-Graduação em Informática na Educação - PPGIE - Universidade \\ Federal do Rio Grande do Sul (UFRGS) - Porto Alegre - RS. \\ ${ }^{3}$ Instituto Federal de Educação, Ciência e Tecnologia Farroupilha - São Borja - RS. \\ \{alexmazzuco,alkrassmann,bgiliane\}@gmail.com,denis.garcia@iffarroupilha.edu.br
}

RESUMO: Este trabalho apresenta o desenvolvimento de um sistema $W e b$ voltado à modelagem molecular tridimensional (3D) que emprega a tecnologia de Realidade Aumentada (RA), auxiliando na compreensão de modelos moleculares e, simultaneamente, criando um repositório digital. Para a realização da pesquisa, utilizouse um método composto por cinco etapas: pesquisa bibliográfica referente aos tópicos condizentes com o sistema; análise e teste das ferramentas computacionais utilizadas; modelagem; desenvolvimento; e aplicação do sistema juntamente aos usuários. Como resultado parcial, observou-se a viabilidade da aplicação do sistema $W e b$ para modelagem molecular $3 \mathrm{D}$ como recurso inovador de ensino voltado à democratização da informação biotecnológica em rede.

Palavras-chave: Democratização, Modelagem Molecular, Realidade Aumentada, Ensino e Aprendizagem.

\section{WEB SYSTEM FOR THREE-DIMENSIONAL MOLECULAR MODELING USING AUGMENTED REALITY AS AN INNOVATIVE TEACHING AND DEMOCRATIZATION OF BIOTECHNOLOGY INFORMATION RESOURCE}

\begin{abstract}
This study presents the development of a Web system towards threedimensional (3D) molecular modeling, that employs Augmented Reality (AR) technology, in order to assist the understanding of molecular models and simultaneously work as a digital repository. To carry out the research it was used a method consisting of five steps as followed: bibliographical research referring to the system topics; analysis and testing of computational tools used; modeling; development; and application of the system with the users. As a partial result, it was observed the feasibility of applying the Web system for 3D molecular modeling as an innovative teaching resource towards the democratization of biotechnology information.
\end{abstract}

Keywords: Democratization, Molecular Modeling, Augmented Reality, Teaching and Learning.

\section{Introdução}

As Tecnologias da Informação e Comunicação (TIC) desempenham, constantemente, um comportamento influente na prática habitual cotidiana da maior 
parte da população, intervindo nos hábitos, nas escolhas e nas formas pelas quais adquirem novos conhecimentos, incluindo, a forma de democratizá-los. Castells (1999), em seu livro "A Sociedade em Rede", declara que a tecnologia da informação está presente em todos os âmbitos da atividade humana e que, por isso, a revolução da tecnologia da informação é o principal ponto para realizar a análise da complexidade da economia, sociedade e cultura em formação.

Nesse contexto, Santos (2012, p. 28) complementa a ideia com a concepção de aceleração tecnológica por acessos geograficamente distintos, afirmando que "o tempo real autoriza usar o mesmo momento a partir de múltiplos lugares; e todos os lugares a partir de um só deles. E, em ambos os casos, de forma concatenada e eficaz.". O autor ainda corrobora em relação ao conhecimento instantâneo, assegurando que "nunca houve antes essa possibilidade oferecida pela técnica à nossa geração de ter em mãos o conhecimento instantâneo do acontecer do outro".

Nesse contexto, observa-se que a utilização das TIC no trabalho pedagógico também vem viabilizando o surgimento de novos paradigmas de aprendizagem e métodos de ensino, bem como auxiliando no processo de democratização de informações biotecnológicas. Destaca-se como um desses novos modelos a utilização da tecnologia de Realidade Aumentada (RA), onde a imagem produzida por dispositivos computacionais permite melhorar a imagem real por meio de informações adicionais, possibilitando a interação em tempo real e controlando ambos os objetos, reais e virtuais (MIHELJ et al., 2013). Herpich et al. (2017) complementam que na RA componentes gráficos gerados por computador são apresentados sobre elementos físicos do mundo, juntamente com o ambiente real.

Diante do exposto, observa-se uma problemática dirigida a dois pontos cruciais. $\mathrm{O}$ primeiro evidencia que devido à complexidade das composições estruturais que inúmeras moléculas possuem, a aprendizagem pode se tornar abstrusa. Esse problema pode estar ligado à intuitividade e à interatividade em relação aos modelos 3D existentes, pois muitos são limitados em realismo de detalhes, e nem sempre são suficientemente intuitivos para que seus usuários possam retirar informações e/ou conclusões com propriedade sobre os mesmos.

O segundo ponto crucial é condizente com a concepção mencionada por Santos (2012, p. 28), de que "a informação instantânea e globalizada por enquanto não é generalizada e veraz porque atualmente é intermediada pelas grandes empresas da informação". Isto é, está disponibilizada em um meio de comunicação, como a Web, contudo, nota-se a ausência de informações instantâneas e fidedignas, e a democratização informacional é limitada pelos interesses corporativos, principalmente no que tange às áreas específicas (científicas) relacionadas à biotecnologia.

Para superar o primeiro ponto mencionado, nesta pesquisa é explorada a possibilidade de utilizar recursos de RA, que transformam informações referentes às estruturas moleculares em modelos virtuais $3 \mathrm{D}$, provendo um ambiente no qual os estudantes podem interagir de uma maneira mais natural, por meio de marcadores (símbolos que podem ser impressos em papel). Já em relação ao segundo ponto, o sistema $W e b$ proposto, além de contemplar uma interface de RA, abrange um sistema online de armazenamento de informações estruturais moleculares tridimensionais, as quais estarão disponibilizadas em arquivos do tipo X3D (padrão aberto para distribuir conteúdo 3D), classificados em categorias, constituindo-se, assim, em um repositório $W e b$ de acesso livre.

Dessa forma, a problemática desta pesquisa justifica-se na necessidade de proporcionar aos estudantes uma melhor visualização das constituições estruturais tridimensionais moleculares, facilitando a aquisição do conhecimento. Também 
justifica-se uma vez que é notória a necessidade de democratização informacional biotecnológica de forma autêntica e imediata em rede.

Portanto, o objetivo deste artigo é apresentar o desenvolvimento de um sistema $W e b$ voltado à modelagem molecular tridimensional, empregando técnicas de Realidade Aumentada, atuando também como repositório online para arquivos do tipo X3D. Para sua realização, utilizou-se um método composto por cinco etapas: pesquisa bibliográfica referente aos tópicos condizentes com o sistema e suas respectivas finalidades, análise e teste das ferramentas computacionais utilizadas no sistema, modelagem, desenvolvimento e aplicação do sistema juntamente aos usuários.

O artigo está estruturado da seguinte forma: na seção 2 são apresentados conceitos relacionados à modelagem molecular, na seção 3 a Realidade Aumentada na educação é abordada e discutida, na seção 4 apresenta-se uma contextualização sobre repositórios digitais e democratização da informação biotecnológica em rede, a seção 5 discorre sobre a concepção e funcionamento do sistema $W e b$, na seção 6 são apresentados procedimentos metodológicos da pesquisa, a seção 7 destina-se a apresentar os resultados parciais de aplicação, finalizando na seção 8 com a conclusões.

\section{Modelagem Molecular}

De acordo com Leach (2001), a modelagem molecular está relacionada às formas de mimetizar o comportamento de moléculas e sistemas moleculares e, invariavelmente, está associada à modelagem por computador. Engloba não apenas a mecânica quântica, mas também mecânica molecular, minimização, simulações, análise conformacional e outros métodos baseados em computador para compreender e prever o comportamento do sistema molecular.

A visualização estrutural molecular é um estágio primordial na realização do trabalho de biólogos estruturais, sendo que inúmeros esforços têm sido empregados nas duas últimas décadas com o intuito de otimizar a metodologia aplicada por especialistas na observação de moléculas (HARRISON et al., 2013). Assim, técnicas computacionais têm revolucionado a modelagem molecular na medida em que a maioria dos cálculos não poderia ser realizada sem a utilização de computadores. Além disso, um aumento na gama de modelos moleculares, como os modelos "stick", "space filling" e CPK é notável. Estes modelos permitem representações tridimensionais das estruturas moleculares e, uma vantagem importante é que eles são interativos, desempenhando, assim, um papel importante tanto no ensino quanto na pesquisa (LEACH, 2001).

Por ser um tópico bastante abstrato, observa-se que a dificuldade de compreensão das estruturas químicas faz parte da realidade de alunos e professores. Isso pode ocorrer devido ao fato de não conseguirem imaginar a estrutura espacial das moléculas que são ensinadas, pois normalmente é utilizada uma representação bidimensional por meio de slides ou do próprio quadro. Caso não se obtenha o entendimento 3D das estruturas químicas, poderá haver dificuldade de entender determinados comportamentos moleculares.

Sendo assim, a geração de modelos visuais 3D de determinada molécula pode contribuir para compreender, explorar e analisar estruturas químicas de forma mais precisa, possibilitando a visualização dos elementos formadores destas estruturas, dos diferentes tipos de ligações entre átomos e seus ângulos e, ainda, de sua superfície.

\section{Realidade Aumentada na Educação}

O ciberespaço é o novo espaço-tempo, tanto da comunicação quanto da informação, sendo um espaço-tempo virtual e de domínio global com duração 
instantânea, abrangendo inclusive a Realidade Virtual (RV) (SANTOS, 2010). A RV possibilita conceber mundos virtuais com particularidades e funcionalidades bastante semelhantes às do mundo real, tornando-os mais próximos dos da realidade, como pode ser observado na exposição de Santos (2003, p. 110), quando afirma que "a meta é permitir que nesse mundo criado artificialmente se possa sofrer, amar, sonhar, além de pensar. Um mundo alternativo (...)". Contudo, ao contrário da RV, que imerge os usuários em um ambiente artificial, a RA permite que o usuário veja objetos virtuais tridimensionais sobrepostos aos objetos do mundo real.

A tecnologia de RA pode ser integrada a inúmeras áreas do conhecimento, como História, Geografia, Artes, Física, Astronomia e, segundo Pasaréti et al. (2011), seu uso pode abranger representações 3D de células em Biologia ou em Química, exibir estruturas moleculares. Assim, é possível observar a existência de vários trabalhos que utilizam esta tecnologia com o potencial de manipular estruturas biomoleculares (BORREL e FOURCHES, 2017), como o Chempreview (ZHENG e WALLER, 2017), que proporciona uma interface baseada em RA para o manuseio de moléculas e o Augmented Chemical Reactions (MAIER, KLINKER e TÖNNIS, 2009), que oferece um ambiente com RA direcionado à visualização de reações químicas entre moléculas.

Entre as inúmeras possibilidades tecnológicas, a RA surge como facilitadora no processo de ensino-aprendizagem (PANEGALLI et al., 2015), proporcionando uma rica experiência educacional e, por se apresentar amplamente interativa, sua utilização pode estimular e motivar os estudantes nas questões acadêmicas (OLIVEIRA e MANZANO, 2016). Essa tecnologia pode auxiliar no entendimento de modelos virtuais, dispostos em determinado espaço, e utilizados como materiais de aprendizagem no mundo real, oportunizando aos usuários uma rápida e profunda experiência de multimídia aumentada (MAITI et al., 2016). Herpich et al. (2017) destacam ainda que a capacidade de visualização de fenômenos não perceptíveis no mundo real em escala, sob diferentes perspectivas e ângulos, leva ao alcance de níveis mais altos de engajamento, permitindo aos estudantes assimilar conceitos abstratos e complexos.

Portanto, a Realidade Aumentada emerge como instrumento tecnológico facilitador na assimilação de conceitos e significados de modelos moleculares, proporcionando maior intuitividade e aumentando a capacidade de interatividade dos estudantes com os mesmos.

\section{Repositório Digital e Democratização da Informação Biotecnológica em Rede}

Segundo Messa et al. (2011, p. 83), "a Internet surgiu e facilitou a comunicação entre as pessoas, conseguindo uni-las de tal forma que nenhum outro meio de comunicação ou tecnologia antes já fizera”. Bilhões de pessoas estão conectadas em todo o planeta e podem participar de qualquer processo de cooperação, pois de acordo com Tapscott (2007, p. 90) "ao contrário de antes, quando custos eram altos, as pessoas podem colaborar e compartilhar as suas criações a um custo muito pequeno".

A Internet permitiu a interconexão da sociedade, viabilizando sua operação "em rede" (CASTELLS, 1999), possibilitando a democratização informacional. Utilizando a Internet como meio para essa democratização, Lévy (1997), considera a rede como um futuro democrático para a humanidade, admitindo a ideia de uma democracia eletrônica. Bossière (2010) corrobora, ao destacar que as TIC concedem uma grande diversidade de novas oportunidades e, graças a uma melhor informação e maior participação, pode-se pensar em uma "democracia eletrônica".

Com o advento da Internet, os repositórios digitais, onde uma gama de recursos é catalogada, organizada e disponibilizada publicamente ou com requisitos de acesso, alastraram-se no âmbito da intitulada sociedade informacional, tornando-se um 
elemento integrador e democrático. Masson (2008, p. 144) afirma que estes repositórios "caracterizam-se pelas tecnologias da informação agindo sobre a informação, seja na produção, coleta, disseminação e preservação da informação, seja alterando as práticas editoriais clássicas, ainda que não através de uma total ruptura". O autor complementa esclarecendo que a lógica das redes de computadores é incorporada ao conceito de repositório digital, que por sua vez se encaixa na morfologia da própria rede. Assim, possibilita a comunicação em grupos de debate da comunidade científica, a interação e a acessibilidade democrática ao conhecimento e aos bancos de dados disponíveis nos repositórios digitais, com a incrementação do acesso livre a uma diversidade de conteúdo.

O campo da biotecnologia apresenta constante crescimento informacional, por envolver uma gama de áreas, como biologia molecular (modelagem molecular), microbiologia, biologia celular, bioquímica, informática, etc. Assim, a biotecnologia assume um papel de tamanha importância, que está relacionada com a transformação do modelo tecnológico. É o que salienta Santos (2003, p. 24): “os especialistas já dispõem de dados suficientes para acreditar que a biotecnologia e a revolução dos novos materiais constituem a próxima onda das altas tecnologias, e que tal tendência conduzirá a uma mudança de paradigma tecnológico".

Dessa forma, percebe-se uma relação direta entre os repositórios digitais e a democratização informacional biotecnológica em rede, os quais, no âmbito da sociedade informacional, evidenciam a crescente demanda pela informação e pela tecnologia. Ainda, é significativo realçar que a utilização de repositórios digitais está sendo cada vez mais ampliada na rede, objetivando o arquivamento digital voltado às mais distintas finalidades, entre elas, o arquivamento de informações biotecnológicas.

\section{Concepção e Funcionamento do Sistema Web}

A concepção do sistema Web enfatiza a modelagem molecular tridimensional, por meio de uma interface de Realidade Aumentada, visando utilizá-lo como recurso inovador de ensino e de democratização da informação biotecnológica em rede, com o intuito de auxiliar no processo de compreensão de modelos moleculares e funcionar como repositório digital na $W e b$.

Com a finalidade de atender a essas duas premissas primordiais, a primeira objetivando o uso da RA como interface para a manipulação de modelos moleculares e a segunda focando na sua utilização como repositório digital, o sistema segmentou-se em duas áreas: Área de Acesso Restrito e Área de Acesso Livre.

A Área de Acesso Restrito exige autenticação, permitido ao usuário com o perfil "Administrador" realizar o gerenciamento de todo o sistema, incluindo o cadastro dos demais usuários com perfis "Professor" e "Aluno". Cada Professor poderá gerar novos "Grupos" (turmas), sendo estes formados por Alunos previamente cadastrados.

O Professor também pode cadastrar "Moléculas", em categorias previamente determinadas, provendo informações detalhadas sobre estas, bem como fazendo upload de seus respectivos arquivos X3D. No momento do registro de uma nova "Aula", o Professor deverá informar qual Grupo fará parte desta aula, incluindo todas as Moléculas que estarão disponíveis para estudo. Logo após, para utilizar a interface de RA em determinada molécula, basta qualquer usuário acessar a seção "Minhas Aulas" e clicar no link "Realidade Aumentada". O estudante terá acesso a todas as aulas às quais seu Professor o vinculou.

Aos Alunos e demais usuários, basta acessar o sistema e navegar pelas categorias (como aminoácidos, proteínas, vírus, etc.) e, encontrando a molécula procurada, clicar no link "Realidade Aumentada" para utilizar a interface de RA. 
Observa-se que para fazer uso desta interface, o usuário deverá possuir um dispositivo (como microcomputador ou notebook) que disponha de uma webcam, bem como um marcador. Dessa forma, no momento em que a imagem do marcador for capturada e reconhecia, será gerado sobre este, o modelo molecular tridimensional desejado (Figura 2).

Além disso, usuários também podem "sugerir" a inclusão e o estudo de uma nova molécula, contribuindo com a ampliação informacional biotecnológica. Para tanto, devem submeter sua sugestão, informando descrições detalhadas sobre a molécula sugerida, juntamente com o respectivo arquivo X3D. Na sequência, a sugestão será encaminhada ao professor, que a avaliará, permitindo ou não sua inclusão no sistema.

A Área de Acesso Livre não exige autenticação, justamente para promover a democratização informacional e funcionar como um repositório digital livre, permitindo acesso a informações biotecnológicas, mais precisamente, referentes às estruturas tridimensionais moleculares.

Observa-se que imagens moleculares tridimensionais, manipuladas por meio de interfaces de RA, usualmente são geradas a partir de arquivos X3D. Por definição de concepção do sistema, determinou-se a utilização de arquivos X3D como essência para a renderização dessas imagens, pois, além do X3D ser um padrão aberto para distribuir conteúdo 3D amplamente utilizado, softwares gratuitos e conceituados no meio científico, voltados à visualização e à modelagem molecular, suportam a exportação de objetos para o padrão X3D. Dessa forma, aproveitam-se os recursos de visualização e modelagem molecular específicos de cada software, permitindo explorar o que há de melhor em cada um deles, gerando um modelo molecular 3D adequado às pretensões de cada usuário, ao exportá-lo para o padrão X3D e, por fim, utilizá-lo no sistema Web.

\section{Procedimentos Metodológicos}

Com o objetivo de elucidar os procedimentos de desenvolvimento deste trabalho, na sequência apresentam-se as cinco fases metodológicas. Até o momento as três primeiras fases dos procedimentos metodológicos foram concluídas, sendo que a quarta etapa encontra-se em processo de execução.

$\mathrm{Na}$ primeira fase foi realizada uma pesquisa bibliográfica, contemplando os tópicos condizentes com o sistema e suas respectivas finalidades, como a modelagem molecular, Realidade Aumentada na educação e, por último, repositório digital e democratização da informação biotecnológica em rede, os quais compõem, respectivamente, as subseções 2,3 e 4 deste artigo.

Na segunda fase foram analisadas e avaliadas, na área da Computação Gráfica, as principais ferramentas e bibliotecas (frameworks) gráficas gratuitas destinadas à geração e manipulação de objetos $3 \mathrm{D}$ na $W e b$, verificando sua utilização como instrumentos de representação dos modelos moleculares tridimensionais. E, ainda, de toolkits de RA para $W e b$, como forma de interação com o modelo obtido, bem como o padrão de arquivos suportado pelo sistema, no caso, X3D.

$\mathrm{Na}$ terceira fase foi realizada a modelagem do sistema $\mathrm{Web}$, contemplando questões condizentes às definições das funcionalidades, do banco de dados, do design e da seleção das estratégias mais adequadas para a integração da interface de Realidade Aumentada ao sistema. Na quarta fase ocorrerá o desenvolvimento do sistema, sendo realizada a implementação dos módulos indispensáveis à sua adequada execução.

$\mathrm{Na}$ quinta fase o sistema $W e b$ será disponibilizado para uso no âmbito educacional, aplicando-o a alunos de uma instituição pública de Ensino Médio, Técnico e Superior em Tecnologia. Na sequência, serão realizadas as avaliações de contexto computacional e educacional. Após a coleta de dados, os mesmos serão analisados de 
forma a avaliar como o sistema influenciou no processo de ensino-aprendizagem de assuntos relacionados às estruturas moleculares, mensurando a efetividade deste.

\section{Resultados Parciais}

Os principais resultados da primeira fase foram apresentada ao longo deste artigo, essencialmente nas seções 2, 3 e 4. Durante a segunda fase, foram realizadas análises e diversos testes para definir qual framework seria utilizado. Pelo fato do $X 3 D O M$, nos testes realizados, ter demonstrado maior flexibilidade e simplicidade na integração com o JSARToolKit, optou-se por dar ênfase em sua utilização. Na terceira fase as atenções foram voltadas especialmente à determinação das funcionalidades do sistema, possibilitando a adequada operacionalização mediante os três perfis de usuário, bem como à definição do layout, com o intuito de atender os requisitos condizentes à responsividade.

Como resultado do andamento da quarta fase, com o início da implementação funcional básica, já é possível acessar algumas seções do sistema, permitindo gerenciar os menus "Grupos", "Usuários", "Moléculas" e "Aulas", sendo que testes relacionados a usuários, categorias, moléculas e aulas que já foram realizados com sucesso. Na Figura 1, observa-se a interface de cadastramento de usuários a partir de um usuário autenticado no sistema com o perfil "Professor".

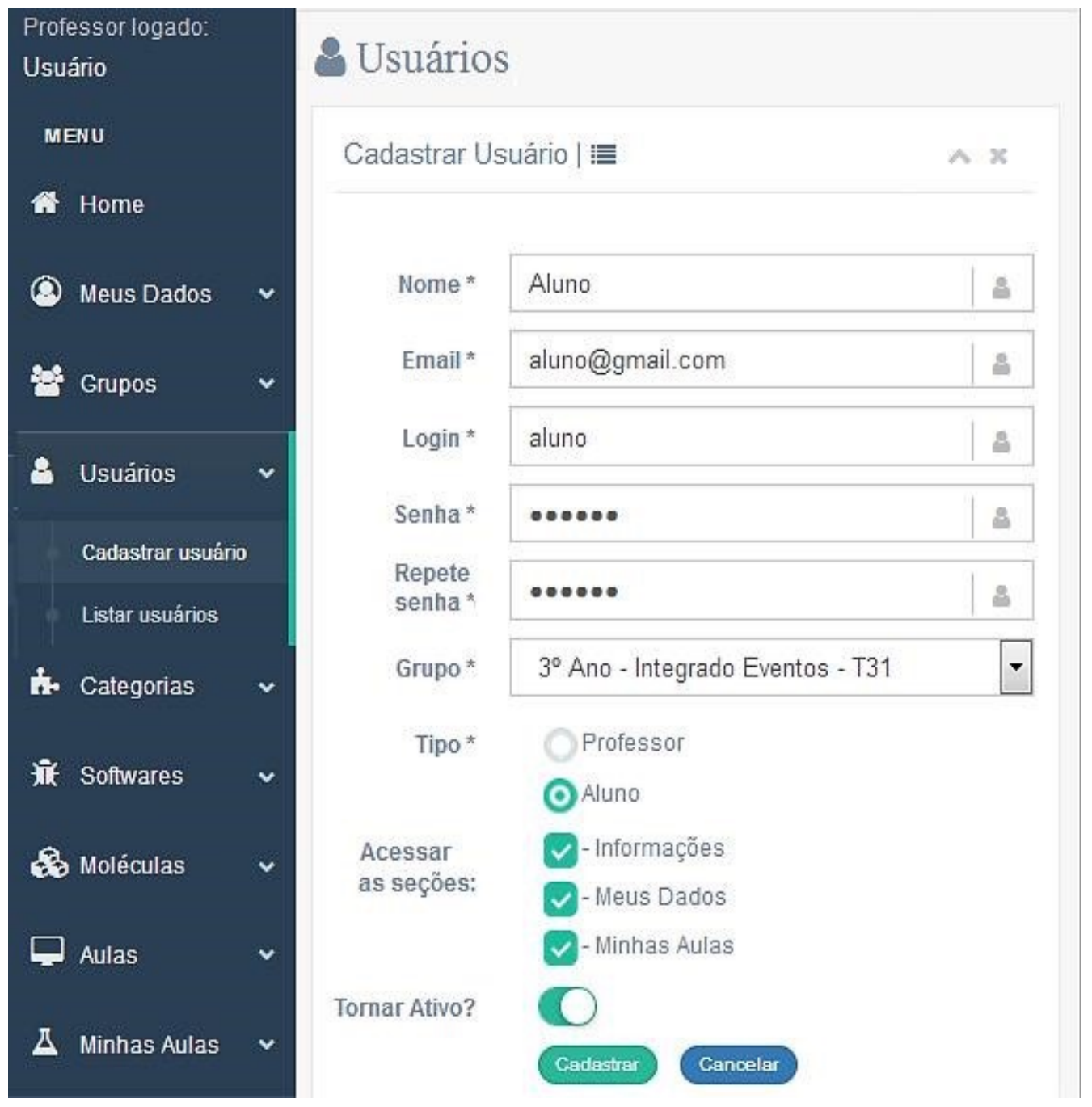

Figura 1 - Interface de cadastro de usuários 
Além disso, foi viabilizada a utilização da interface de Realidade Aumentada, na seção "Minhas Aulas", como pode ser observado no protótipo (Figura 2).

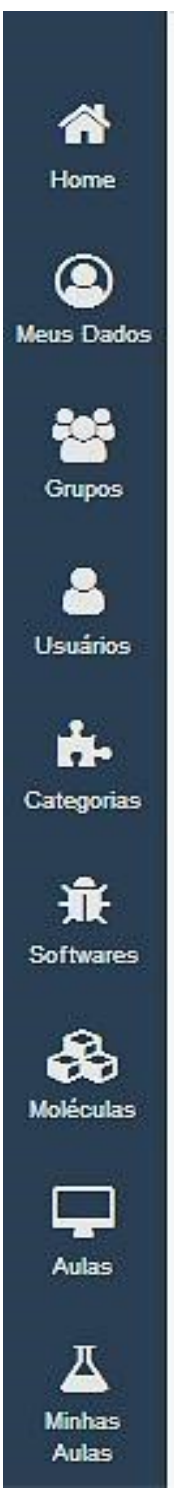

\section{匹 Minhas Aulas}

\section{Minhas Aulas}

$\wedge<$

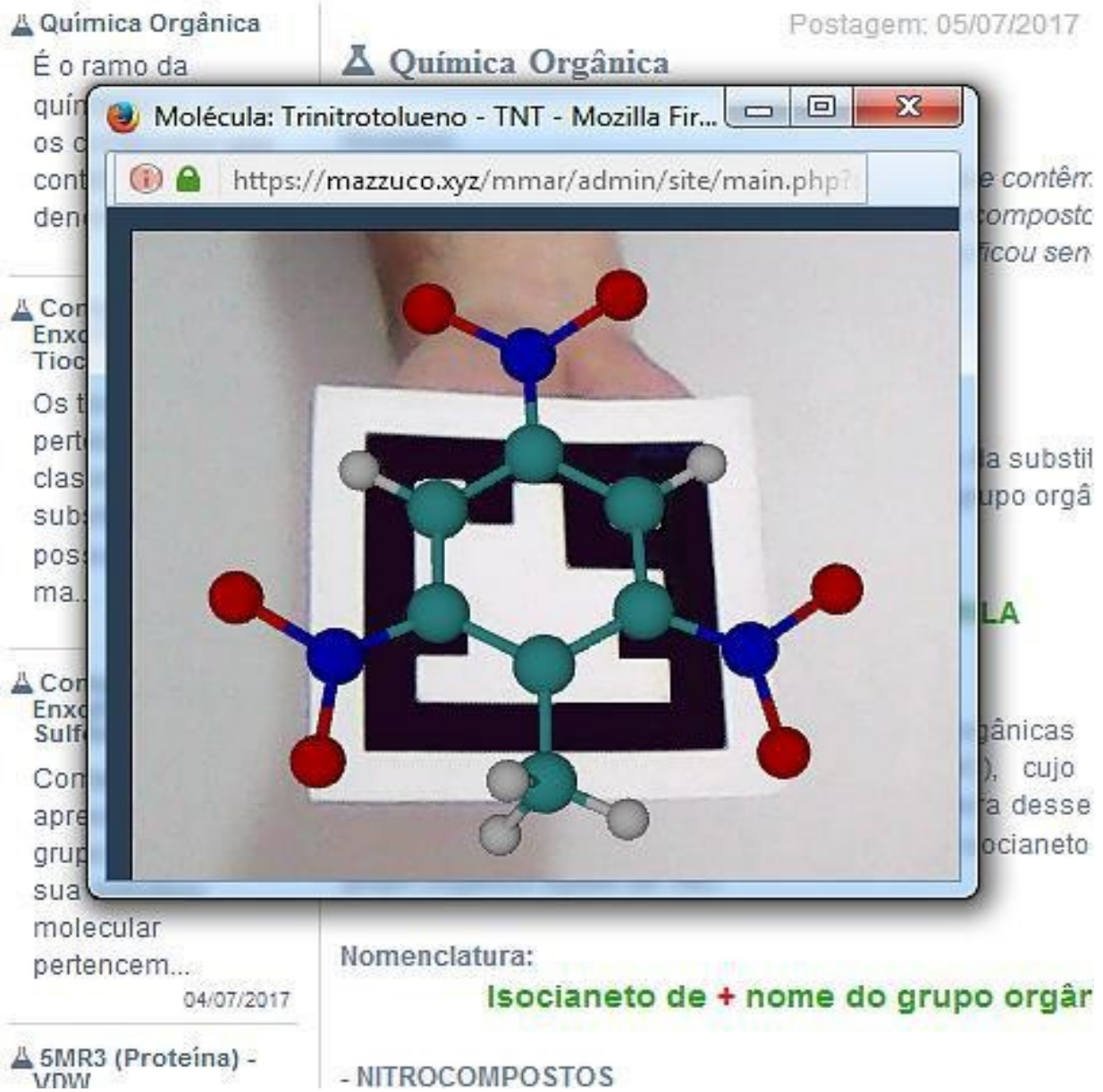

Figura 2 - Utilização da interface de Realidade Aumentada (protótipo)

Em relação ao repositório digital, este se encontra em um processo inicial de implementação, sendo que a interface gráfica (layout) ainda não foi definida. Contudo, esse processo será mais ágil, pois o banco de dados já está modelado e diversas funcionalidades que estão concluídas serão reutilizadas.

\section{Considerações Finais}

É possível perceber que a utilização das Tecnologias da Informação e Comunicação no âmbito do trabalho pedagógico viabilizou o surgimento de novos paradigmas de aprendizagem, modificando o cenário de ensino tradicional, bem como alavancou a democratização de informações biotecnológicas, permitindo a disseminação desse conhecimento.

Entre esses novos modelos pedagógicos destaca-se a utilização da Realidade Aumentada como ferramenta que permite a modelagem molecular tridimensional e 
pode se integrar ao conceito de repositório digital, como meio de popularizar informações referentes a essa área.

O sistema $\mathrm{Web}$ com as características peculiares apresentadas neste artigo possui o potencial de ser considerado inovador e se encontra em pleno desenvolvimento. Ainda estão sendo analisados quais aspectos serão avaliados na sua aplicação, contudo, já se considera analisar as possíveis influências relacionadas à interatividade, à usabilidade e à motivação, proporcionadas pelo sistema.

Para a retenção do feedback do público-alvo, condizentes a estes aspectos, certas metodologias e alguns autores estão sendo estudados, como: as diretrizes relacionadas à interatividade propostas por Silveira e Carneiro (2012); o modelo de questionário referente à usabilidade proposto por Rezende (2013); o modelo de questionário relativo à motivação sugerido por Savi (2011). No que tange ao procedimento de coleta de dados, considera-se utilizar estratégias fundamentadas nos conceitos da abordagem de Likert (1932), conhecidos como escala Likert.

Com sua finalização, pretende-se auxiliar o processo de democratização informacional biotecnológica em rede, disponibilizando informações moleculares 3D em arquivos do tipo X3D, classificados em categorias, constituindo-se, assim, em um repositório $W e b$ de acesso livre. E ainda, almeja-se tornar a interface de RA um instrumento tecnológico inovador e facilitador do processo de compreensão de modelos moleculares, proporcionando um cenário com maior intuitividade e, simultaneamente, aumentando a capacidade de interatividade dos estudantes com os mesmos.

\section{Referências Bibliográficas}

BORREL, A.; FOURCHES, D. RealityConvert: a tool for preparing 3D models of biochemical structures for augmented and virtual reality. Bioinformatics, v. 33, n. 23, p. 3816-3818, dez. 2017. Disponível em: <https://doi.org/10.1093/bio informatics/btx 485>. Acesso em: 17 jul. 2018.

BOSSIÈRE, M. Democracia Electrónica: ¿Qué Desafíos para América Latina? Santiago de Chile: Editorial Aún Creemos en Los Sueños, 2010. 76 p.

CASTELlS, M. A Sociedade em Rede - A Era da Informação: Economia, Sociedade e Cultura, vol. 1. São Paulo: Paz e Terra, 1999. 698 p.

HARRISON, K. et al. Electronic visualisation in chemistry: From alchemy to art. In: Conference Proceedings, Electronic Workshops in Computing (eWiC), British Computer Society, London, p. 267-274, jul. 2013. Disponível em: $<$ https://arxiv.org/ abs/1307.6360>. Acesso em: 19 maio 2017.

HERPICH, F. et al. A Comparative Analysis of Augmented Reality Frameworks Aimed at the Development of Educational Applications. Creative Education, 8(09), 1433, 2017.

LEACH, A. R. Molecular Modelling: Principles and Applications. Prentice Hall, 2001. $744 \mathrm{p}$.

LIKERT, R. A technique for the measurement of attitudes. Archives of Psychology, v. 140, p. 1-55, 1932.

LÉVY, P. O que é o virtual? São Paulo: Ed. 34, 1997. 124 p. 
MAIER, P.; KLINKER, G; TÖNNIS, M. Augmented Reality for teaching spatial relations. Conference of the International Journal of Arts \& Sciences, maio, 2009.

MAITI, A. et al. Key aspects of integrating augmented reality tools into peer-to-peer remote laboratory user interfaces. IEEE 13th International Conference on Remote Engineering and Virtual Instrumentation (REV), Madrid, p. 16-23, fev. 2016.

MASSON, S. M. Os repositórios digitais no âmbito da sociedade informacional. PRISMA.COM - Rev. de Ciência da Informação e da Comunicação do CETAC, Porto, n. 7, p. 105-152, 2008.

MESSA, A. F. et al. Sustentabilidade Ambiental e os Novos Desafios na Era Digital: estudos em homenagem a Benedito Guimarães Aguiar Neto. São Paulo: Saraiva, 2011. $500 \mathrm{p}$.

MIHELJ, M. et al. Virtual Reality Technology and Applications. Springer Science \& Business Media, 2013. 241 p.

OLIVEIRA, L. D. de; MANZANO, R. C. Aplicações de realidade aumentada no ensino de Física a partir do software LAYAR. RENOTE, v. 14, n. 1, 2016.

PANEGALLI, F. S. et al. Realidade Aumentada no Desenvolvimento de Jogos Educacionais: Um Estudo de Caso de um Jogo de Língua Inglesa. RENOTE, v. 13, n. $1,2015$.

PASARÉTI O. et al. Augmented Reality in education, INFODIDACT, Informatika Szakmódszertani Konferencia, 2011.

REZENDE, C. S. Modelo de Avaliação de Qualidade de Software Educacional para o Ensino de Ciências. 2013. 131 p. Dissertação de Mestrado (Programa de Pós-Graduação em Ensino de Ciências) - Universidade Federal de Itajubá, Itajubá, MG, 2013.

SANTOS, B. S. A Gramática do Tempo: Para uma Nova Cultura Política, 3.ed. São Paulo: Cortez, 2010. 511 p.

SANTOS, L. G. Politizar as Novas Tecnologias: O Impacto Sócio-Técnico da Informação Digital e Genética. São Paulo: Editora 34, 2003. 320 p.

SANTOS, M. Por Uma Outra Globalização: Do Pensamento Único à Consciência Universal, 22.ed. Rio de Janeiro: Record, 2012.

SAVI, R. Avaliação de Jogos para a Disseminação do Conhecimento, no estado de Santa Catarina. Florianópolis: UFSC, 2011. 236 p. Tese de doutorado.

SILVEIRA, M. S.; CARNEIRO M. L. F. Diretrizes para a Avaliação da Usabilidade de Objetos de Aprendizagem. In: Simpósio Brasileiro de Informática na Educação (SBIE). 2012, Rio de Janeiro. Anais. Disponível em: http://br-ie.org/pub/index.php/sbi e/article/view/1713/1474. Acessado em: 25 maio 2017.

TAPSCOTT, D.; WILLIAMS, A. D. Wikinomics: Como a Colaboração em Massa Pode Mudar o Seu Negócio. Rio de Janeiro: Nova Fronteira, 2007. 368 p.

ZHENG, M.; WALLER, M. P. ChemPreview: an augmented reality-based molecular interface. Journal of Molecular Graphics and Modelling, v. 73, p. 18-23, 2017. 